University of Wollongong

Research Online

Australian Institute for Innovative Materials -

Papers

Australian Institute for Innovative Materials

$1-1-2017$

\title{
The origin of enhanced photocatalytic activities of hydrogenated TiO2
} nanoparticles

Yani Liu

Beihang University

Haifeng Feng

University of Wollongong, Beihang University, hf533@uowmail.edu.au

Xiaobing Yan

Hebei University

Jiaou Wang

Chinese Academy Of Sciences

Huagui Yang

East China University of Science and Technology

See next page for additional authors

Follow this and additional works at: https://ro.uow.edu.au/aiimpapers

Part of the Engineering Commons, and the Physical Sciences and Mathematics Commons

Research Online is the open access institutional repository for the University of Wollongong. For further information contact the UOW Library: research-pubs@uow.edu.au 


\title{
The origin of enhanced photocatalytic activities of hydrogenated TiO2 nanoparticles
}

\author{
Abstract \\ The photocatalytic activity of semiconductors is largely governed by their light absorption, separation of \\ photoinduced charge carriers and surface catalytically active sites, which are primarily controlled by the \\ morphology, crystalline size, structure, and especially the electronic structure of photocatalysts. Black TiO \\ 2 is recognized as one of the most promising visible-light photocatalysts, due to its significantly enhanced \\ visible-light photocatalytic performance in comparison to intrinsic TiO 2 . In this work, black TiO 2 is \\ synthesized through the hydrogenation process. The sample shows a TiO 2 @TiO 2-x core/shell structure \\ which is attributed to hydrogenation. By using X-ray photoelectron spectroscopy (XPS), X-ray absorption \\ spectroscopy (XAS), and nuclear magnetic resonance (NMR) spectroscopy, we identified the featured \\ midgap electronic states in black TiO 2 , which gave rise to the TiO 2-x shell layer. These states lead to the \\ improvement of visible-light absorption and the separation of photoinduced charge carriers, which \\ consequently result in remarkable enhanced photocatalytic activities in black TiO 2 .

\section{Disciplines \\ Engineering | Physical Sciences and Mathematics}

\section{Publication Details} \\ Liu, Y., Feng, H., Yan, X., Wang, J., Yang, H., Du, Y. \& Hao, W. (2017). The origin of enhanced photocatalytic \\ activities of hydrogenated TiO2 nanoparticles. Dalton Transactions: an international journal of inorganic \\ chemistry, 46 (32), 10694-10699.
}

\section{Authors}

Yani Liu, Haifeng Feng, Xiaobing Yan, Jiaou Wang, Huagui Yang, Yi Du, and Weichang Hao 


\section{The Origin of Enhanced Photocatalytic Activities of}

\section{Hydrogenated $\mathrm{TiO}_{2}$ nanoparticles}

Yani Liu ${ }^{\mathrm{a}, \mathrm{f}}$, Haifeng Feng ${ }^{\mathrm{b}, \mathrm{f}}$, Xiaobing Yan*c ${ }^{\mathrm{c}}$, Jiaou Wang ${ }^{\mathrm{d}}$, Huagui Yang ${ }^{\mathrm{e}}$, Yi Du ${ }^{\mathrm{a}, \mathrm{b}, \mathrm{f}}$, Weichang $\mathrm{Hao}^{* \mathrm{a}, \mathrm{b}, \mathrm{f}}$

a Department of Physics and Key Laboratory of Micro-Nano Measurement, Manipulation and Physics, Ministry of Education, Beihang University, Beijing 100191, China

${ }^{\mathrm{b}}$ Institute for Superconducting and Electronic Materials, University of Wollongong, Wollongong, NSW 2500, Australia

${ }^{\mathrm{c}}$ College of Electronic and information Engineering, Hebei University, Baoding 071002, China

${ }^{d}$ Institute of High Energy Physics, Chinese Academy of Sciences, Beijing, 10049, China

${ }^{\mathrm{e}}$ School of Materials Science and Engineering, East China University of Science and Technology, Shanghai 200237, China

f BUAA-UOW Joint Research Center, Beihang University, Beijing 100191, China 


\begin{abstract}
Photocatalytic activity of semiconductors are largely governed by their light absorption, separation of photoinduced charge carriers and surface catalytic active sites, which are primarily controlled by the morphology, crystalline size and structure, and especially the electronic structure of photocatalysts. Black $\mathrm{TiO}_{2}$ is recognized as one of the most promising visible-light photocatalysts, due to its significantly enhanced visible-light photocatalytic performance in comparison to intrinsic $\mathrm{TiO}_{2}$. In this work, black $\mathrm{TiO}_{2}$ is synthesized through the hydrogenation process. The sample shows a $\mathrm{TiO}_{2} @ \mathrm{TiO}_{2-\mathrm{x}}$ core/shell structure which is attributed to hydrogenation. By using X-ray photoelectron spectroscopy (XPS), X-ray absorption spectroscopy (XAS), and nuclear magnetic resonance (NMR), we identified the featured midgap electronic states in black $\mathrm{TiO}_{2}$, which is given rise to the $\mathrm{TiO}_{2-x}$ shell layer. These states lead to the improvement of visible-light absorption and the separation of photoinduced charge carriers, which consequently result in the remarkable enhanced photocatalytic activities in black $\mathrm{TiO}_{2}$.
\end{abstract}




\section{Introduction}

Hydrogen production from overall water splitting using semiconductor photocatalysts is one of the most promising routes to harvest solar energy. ${ }^{1} \mathrm{TiO}_{2}$ has attracted substantial interest as a photocatalyst due to its excellent activity for water splitting under ultraviolet (UV) light irradiation., ${ }^{2,3}$ To obtain higher efficiency, much effort has been made to enable its absorption of visible light for enhanced photocatalytic activity, with metal, ${ }^{4}$ non-metal, ${ }^{5,6}$ or self-doping. ${ }^{7,8}$ It has triggered intense research interests since Chen et al. ${ }^{9}$ reported the black $\mathrm{TiO}_{2}$ nanoparticles with strong visible-light absorption and outstanding visible-light photocatalytic activities. Since then, persistent efforts have been made in both experimental and theoretical studies on black $\mathrm{TiO}_{2}{ }^{10-15}$

However, origin and the mechanism for the enhanced photocatalytic activities in the black $\mathrm{TiO}_{2}$ remain unclear. The link between photocatalytic performance and electronic structures are also missing. This is because of technical difficulties in detecting fine electronic states of the surface layer, where photocatalytic process occurs. In addition, various black $\mathrm{TiO}_{2}$ has been fabricated through a variety of methods, including high pressure treatment, ${ }^{11}$ high temperature treatment, ${ }^{12}$ long time hydrogenation, ${ }^{13}$ plasma assisted hydrogenation ${ }^{14}$ and hydrogenation of Ni-doped $\mathrm{TiO}_{2}{ }^{15}$. Although all of them present black color, the surface electronic states are expected to be distinct to each other.

In this paper, a facile and economical method for preparing hydrogenated $\mathrm{TiO}_{2}$ with hydrogenated disorders in the surface layer surrounding a crystalline core is adopted. According to Sun et al., the optimal $\mathrm{H}_{2}$ production under $\mathrm{UV}$-Vis irradiation is up to $6.5 \mathrm{mmol} \mathrm{h}^{-1} \mathrm{~g}^{-1}$, which is about 7.2 times more than that of the original $\mathrm{TiO}_{2}{ }^{16}$ 
Methods combining XPS, UPS and XAS were used to systematically characterize and analysis the fine electronic states, including valence band (VB), midgap states and conduction band $(\mathrm{CB})$, which determine the absorption of light, charge separation and surface catalytic reactions of black $\mathrm{TiO}_{2} \cdot{ }^{17,18,19}$ In this work, it is found that the $\mathrm{TiO}_{2} @ \mathrm{TiO}_{2-\mathrm{x}}$ core/shell structure were formed after hydrogenation process. The midgap states forms due to $\mathrm{TiO}_{2-\mathrm{x}}$ surface layer, which promtevisible-light absorption and separation of photoinduced charge carriers. The core-shell structure and surface disorder layer effectively facilitate the enhancement in visible-light photocatalytic activity of hydrogenated $\mathrm{TiO}_{2}$.

\section{Experimental section}

\section{Syntheses of the hydrogenated $\mathrm{TiO}_{2}$}

P25 was commercial available Degussa P25 $\mathrm{TiO}_{2}$ nanopowder. Anatase $(99.8 \%, 25$ $\mathrm{nm})$, rutile $(99.8 \%, 25 \mathrm{~nm})$ and $\mathrm{NaBH}_{4}(98 \%)$ were purchased from Alfa Reagent Company. Ethanol was purchased from Beijing Chemical Reagent Company and used as received without any further purification.

$4.0 \mathrm{~g}$ of $\mathrm{TiO}_{2}$ nanoparticle powder was mixed with $1.5 \mathrm{~g}$ of $\mathrm{NaBH}_{4}$ and then stirred for 30 min thoroughly at room temperature. Then the mixture was transferred into a porcelain boat, placed in a tubular furnace, sintered from room temperature to $300{ }^{\circ} \mathrm{C}$ under an Ar atmosphere (at a heating rate of $10{ }^{\circ} \mathrm{C} \mathrm{min}^{-1}$ ) and then treated for 20, 40, 60, 80 min respectively. After naturally cooling down to the room temperature, different degrees of hydrogenated $\mathrm{TiO}_{2}$ were obtained, simply washed with deionized water and ethanol several times to remove untreated $\mathrm{NaBH}_{4}$, and dried at $80{ }^{\circ} \mathrm{C}$.

\section{Characterization of the hydrogenated $\mathrm{TiO}_{2}$}


The HRTEM images were taken on a Phillips CM 200 microscope. The crystalline structure was recorded by an X-ray diffractometer (XRD) (Bruker AXS D8 Focus), with $\mathrm{Cu} \mathrm{K} \alpha$ radiation $(\lambda=1.54 \AA$ ). The ultraviolet-visible diffuse reflectance (UV-Vis) spectra were collected on a Cintra-10e spectrometer using $\mathrm{BaSO}_{4}$ as reference sample. XPS spectra were performed on an ESCALABMKII spectrometer with an Al-Ka $(1486.6 \mathrm{eV})$ achromatic X-ray source. XAS spectra were conducted at the Photoelectron Spectroscopy Station (Beamline 4W9B) of the Beijing Synchrotron Radiation Facility of the Institute of High Energy Physics, Chinese Academy of Sciences. The NMR studies are carried out on 700MHz Agilent NMR spectrometers at a magnetic field strength of $16.4 \mathrm{~T}$. For the surface photovoltage (SPV) spectrum measurement, we used a piece of blank conductive ITO glass as the counter electrode. The positive electrode of the lock-in input is always connected to the pole on the light incident side of the sandwich-like structure of ITO (indium tin oxide)-sample-ITO.

\section{Results and Discussion}

Fig. 1 shows the TEM and HRTEM images of the $\mathrm{TiO}_{2}$ nanocrystals before and after $\mathrm{NaBH}_{4}$ treatment. No difference can be found in their particle sizes and the lattice plane distance $[0.35 \mathrm{~nm}$ for anatase (101)] in the crystalline core.. For the Fig. 1c, a 1-2 $\mathrm{nm}$ thickness disordered layer appears in the surface of hydrogenated nanoparticles with reduction-treatment at $300{ }^{\circ} \mathrm{C}$ for $40 \mathrm{~min}$. When the treatment time is raised to $80 \mathrm{~min}$ (Fig. 1d), the disordered layer becomes thicker and is clearer to be observed relative to the pristine $\mathrm{TiO}_{2}$ (Fig. 1b). From the XRD patterns (Fig. S1 in Supporting Information), we find that both the intensity of main peaks for anatase and rutile weaken and broaden with the increase of the treatment time, which is correspond with increase of the thickness of the disordered layer. The TEM results clearly reveal that the hydrogenated sample has a $\mathrm{TiO}_{2} @ \mathrm{TiO}_{2-\mathrm{x}}$ core/shell structure. 
UV-Vis absorption spectra of the as-prepared $\mathrm{TiO}_{2}$ are shown in Fig. 2. They show that the absorption region broadens from $400 \mathrm{~nm}$ to the near infrared region with the increase of reaction time. Meanwhile, the intensity of the visible-light absorption band gradually increases, which is consistent with the change of the color from white to black (inset of Fig. 2). The appearance and increase of visible-light absorption originate from the impurity deep and shallow energy levels in hydrogenated $\mathrm{TiO}_{2}$ (Fig. S2 in Supporting Information). ${ }^{20}$ Impurity band cannot narrow the bandgap but provide a channel in the midgap for visible-light absorption. In our result as shown in Fig. 2, the absorption edges of hydrogenated samples have slight red shifts by contrast with the raw $\mathrm{TiO}_{2}$, which reflects that the band gaps of hydrogenated $\mathrm{TiO}_{2}$ are narrowed slightly and their intrinsic absorption change non-significantly.

XPS is used to investigate the change of surface chemical bonding as well as the electronic VB position due to hydrogenation in black $\mathrm{TiO}_{2}$. As shown in the inset of Fig. $3 \mathrm{~b}$, the Ti $2 p_{3 / 2}$ and Ti $2 p_{1 / 2}$ peaks centered at binding energies $458.77 \mathrm{eV}$ and $464.52 \mathrm{eV}$, which are typical characteristics of the $\mathrm{Ti}^{4+}-\mathrm{O}$ bonds in $\mathrm{TiO}_{2}{ }^{21}$ For the hydrogenated samples, these peaks shift to lower binding energies of $458.62 \mathrm{eV}$ and $464.37 \mathrm{eV}$, respectively. It indicates that $\mathrm{Ti}^{3+}$ may form and attribute to the surface Ti-H bonds. ${ }^{22}$ The zone-selective electronic spectroscopic can provide useful information on chemical binding and valence band position on the sample surfaces. ${ }^{23}$ After peak area normalization and subtracting P25 spectra from spectra named 40 min sample, the polarization peak and bulk valley are obtained. The bulk valley at 459.17 $\mathrm{eV}$ corresponds to the $\mathrm{Ti} 2 p_{3 / 2}$ (Fig. 3b) and at $530.32 \mathrm{eV}$ to the $\mathrm{O} 1 s$ (Fig. 3c) in the bulk $\mathrm{TiO}_{2}$. The polarization peak attributes to the bond order-length-strength and nonbonding electron polarization (Fig. S3 in Supporting Information) notation. ${ }^{22}$ As shown in the schematic diagram (Fig. 3a), the atomic undercoordination is formed by 
hydrogenation, which shortens and strengthens the local bond, and deepens the local potential well. The "entrapment state" is then produced with the densified and entrapped bonding electrons. At the same time, the densely entrapped electrons polarize the nonbonding electrons to form the shifting up "polarized state" (Fig. S3 in Supporting Information). The entrapment and polarization, thus, mediate the bandgap, the electron affinity and the Femi level. In the present case, polarization state at $458.27 \mathrm{eV}$ for the $\mathrm{Ti} 2 p_{3 / 2}$ (Fig. 3b) and at $529.27 \mathrm{eV}$ for O $1 s$ (Fig. 3c) shift up to the Femi level comparing with the bulk valley state. The upward polarization state makes the occupied state shifting up to the Femi level. This electronic structure variation happens when the $\mathrm{H}$ atoms enter into the octahedral crystalline and create $\mathrm{Ti}$ undercoordination states (Ti-H bonds or oxygen vacancies). In addition, as shown in the VB spectra (Fig. 3d), the VB edge of hydrogenated sample does not exhibit strong variation, and the VB tail is absent compared with that of the origin sample. It agrees well with previous work that the hydrogen treatment has a negligible effect on VB position of the $\mathrm{TiO}_{2}$ surface. ${ }^{13}$ Our XPS result indicates that the intrinsic electronic state in the bulk $\mathrm{TiO}_{2}$ does not change apparently through the hydrogenation. The impurity electronic state mainly accounts for the midgap energy level.

XAS is a powerful method to investigate the local coordination environment and the midgap energy state. In the schematic diagram of energy level in $\mathrm{TiO}_{2}$ (Fig. 4a), the Ti L-edge XAS consists of contributions from $\mathrm{L}_{3}\left(2 p_{3 / 2}-3 d\right)(457-462 \mathrm{eV})$ and $\mathrm{L}_{2}$ $\left(2 p_{1 / 2}-3 d\right)(462-467 \mathrm{eV})$ transitions. The $t_{2 \mathrm{~g}}$ and $e_{\mathrm{g}}$ peak of $\mathrm{L}_{3}$ refer to the excitation process from Ti $2 p_{3 / 2}$ to $t_{2 \mathrm{~g}}$ and $e_{\mathrm{g}}$ of $\mathrm{Ti} 3 d$ band split by the octahedral crystal field, respectively. ${ }^{24}$ In the similar way, $t_{2 \mathrm{~g}}$ and $e_{\mathrm{g}}$ peak of $\mathrm{L}_{2}$ refer to the excitation process from Ti $2 p_{1 / 2}$ to $t_{2 \mathrm{~g}}$ and $e_{\mathrm{g}}$ of $\mathrm{Ti} 3 d^{25}$ The absorption edges are $457.1 \mathrm{eV}$ in the Ti-L edge of XAS (Fig. 4b) for both the raw and hydrogenated $\mathrm{TiO}_{2}$. In order to analyze 
the change of electronic state, we further perform peak area fitting. Through peak area analysis (Fig. 4c), the $e_{\mathrm{g}}$ peak ratio of $\mathrm{L}_{3}$ and $\mathrm{L}_{2}$ area of the treated $\mathrm{TiO}_{2}$ (hydrogenated for $40 \mathrm{~min}$ ) decrease, while the $t_{2 \mathrm{~g}}$ peak ratio of $\mathrm{L}_{3}$ and $\mathrm{L}_{2}$ increase compared with the pristine $\mathrm{TiO}_{2}$. The two pre-leading peaks marked with asterisks are considerable close to the $\mathrm{CB}$ minimum as they are $0.8 \mathrm{eV}$ and $1.4 \mathrm{eV}$ below the absorption edges, which come from the excitation from $\mathrm{Ti} 2 p_{3 / 2}$ to the continuous density of vacancy states named $V_{1}$ and $V_{2}$ (Fig. 4a). These midgap states broaden the optical absorption in the visible region. ${ }^{26}$ The two pre-leading peaks are believed to relate to the defect states, such as vacancy states and Ti undercoordination state. The higher $t_{2 \mathrm{~g}}$ peak area ratio in the hydrogenated sample reflects its increased defect state because the $t_{2 \mathrm{~g}}$ peak can be highly influenced by two pre-leading peaks. ${ }^{27}$ In addition, oxygen vacancies in $\mathrm{TiO}_{2}$ are known to be shallow donors with the low formation energy, ${ }^{28}$ which were also confirmed by infrared absorption coefficient spectrum. ${ }^{29}$ Those vacancies, Ti-H bonds and Ti undercoordination, which come from the surface disorder layer of $\mathrm{TiO}_{2} @ \mathrm{TiO}_{2-x}$ nanocrystals, therefore, are demonstrated to contribute to the defect states.

As analyzed above, hydrogen induces a disordered layer on the surface of crystalline core. In order to reveal incorporation state of hydrogen, we carried out NMR measurements on the as-prepared samples. Fig. 5 shows the ${ }^{1} \mathrm{H}$ NMR spectra for the untreated and hydrogenated $\mathrm{TiO}_{2}$ nanoparticles. The intensity of the two ${ }^{1} \mathrm{H}$ NMR resonance peaks at $0.45 \mathrm{ppm}$ and $0.8 \mathrm{ppm}$ increase with the treated time extending, while the two peaks are not collected for the untreated $\mathrm{TiO}_{2}$. It suggests that the hydrogen has been successfully introduced to the surface shell of the hydrogenated sample. These two ${ }^{1} \mathrm{H}$ NMR resonance peaks also were observed by Chen et al. ${ }^{30}$ The hydrogen in the shell is expected to diffuse rapidly around the surface layer and lead 
to crystalline structure distortion, more defects formation, which induce the continuous density of vacancy states. Generally, the peak of chemical shift at 4.25-5.3 ppm in Fig. 5 is attributed to the O-H group adsorbed on the surface of material. ${ }^{13}$ It is found that this peak shifts toward the low chemical shift (high field) direction, which is caused by weaker $\mathrm{O}-\mathrm{H}$ bonds with the treatment time increase. The crystal lattice structure is broken and distorted when hydrogen was introduced in the surface of $\mathrm{TiO}_{2}$ nanoparticles. The interaction between this disorder layer and adsorbed $\mathrm{O}-\mathrm{H}$ group on the surface of $\mathrm{TiO}_{2}$ leads to the weaker O-H bonds which may be beneficial to the catalytic decomposition of water.

Beside the characterization of the crystalline structure distortion and electronic structure variation with incorporation state of hydrogen, we utilized the surface photovoltage (SPV) to verify the change of charge separation efficiency before and after the hydrogenation. Fig. 6 exhibits the SPV spectra of pristine $\mathrm{TiO}_{2}$ and hydrogenated $\mathrm{TiO}_{2}(40 \mathrm{~min})$. A broad peak appears in the UV and visible light region (387-407 nm). It indicates that the treated material possesses a better response to light absorption and more effective photoinduced electron-hole pairs separation, which match with the UV-Vis spectra.

In this study, NMR spectra and HRTEM images clearly show that the core/shell structure $\mathrm{TiO}_{2} @ \mathrm{TiO}_{2-\mathrm{x}}$ successfully formed when hydrogen was introduced into the surface of nanoparticles during hydrogenation process. The XPS and XAS indicate that the shallow energy level from surface states are introduced by inevitable surface oxygen vacancies, hydrogen impurities, $\mathrm{Ti}-\mathrm{H}$ bonds and $\mathrm{Ti}$ undercoordination in the surface shell layer of nanocrystals. Increase of the visible light absorption and the acceleration of separation of photoinduced charge carriers are confirmed to originate from the midgap states that induced by the disorder layer. Therefore, as an effective 
and facile method, hydrogenation under low temperature and pressure can be widely applied in various metal oxide materials.

\section{Conclusion}

In summary, we carried out characterizations and analysis of HRTEM, UV-Vis spectra, XPS, XAS, and VB spectroscopy, NMR and SPV for the hydrogenated and untreated $\mathrm{TiO}_{2}$ nanoparticles. The incorporation of hydrogen brings out a disorder shell layer, which is clearly observed by the HRTEM images and confirmed by the NMR spectra. The midgap states $0.8 \mathrm{eV}$ and $1.4 \mathrm{eV}$ below the $\mathrm{CB}$ minimum are induced by the disorder defect layer, which are demonstrated by the XPS and XAS study. These two shallow levels increase the visible light absorption and accelerate the photoinduced charge separation for the hydrogenated sample. In short, hydrogenation treatment for $\mathrm{TiO}_{2}$ nanoparticles distorts the octahedral crystalline lattice, modify the electronic state, accelerate the separation of photoinduced charge carriers under visible light irradiation. This work is expected to provide a in-depth understanding of the origin of the enhanced photocatalytic activities of hydrogenated $\mathrm{TiO}_{2}$ nanoparticles, and a renewed research perspective for other hydrogenated materials.

\section{Acknowledgements}

The authors are grateful for financial support from the National Natural Science Foundation of China (51472016, 51672018), the Fundamental Research Funds for the

Central Universities (YWF-16-JCTD-B-03, YWF-16--BJ-J-44). H. F. F. and Y. D. thank the Australian Research Council (ARC) for partial support of this work through a Discovery Project (DP140102581, DP170101467). 


\section{References}

1 A. Fujishima, K. Honda, Nature, 1972, 238, 37-38.

2 D. A. Tryk, A. Fujishima, K. Honda, Electrochimica Acta, 2000, 45(15), 2363-2376.

3 E. M. Samsudin, S. B. A. Hamid, J. C. Juan, W. J. Basirun and A. E. Kandjani, Applied Surface Science, 2015, 359, 883-896.

4 W. Choi, A. Termin, M. R. Hoffmann, Angewandte Chemie International Edition in English, 1994, 33(10), 1091-1092.

5 G. Liu, Y. Zhao, C. Sun, F. Li, G. Q. Lu, H. M. Cheng. Angewandte Chemie International Edition, 2008, 47(24), 4516-4520.

6 X. Chen, C. Burda, Journal of the American Chemical Society, 2008, 130(15), 5018-5019.

7 F. Zuo, L.Wang, T. Wu, Z. Zhang, D. Borchardt, P. Feng. Journal of the American Chemical Society, 2010, 132(34), 11856-11857.

8 G. Liu, L. Wang, H. G. Yang, H. M. Cheng, G. Q. Lu, Journal of Materials Chemistry, 2010, 20(5), 831-843.

9 X. Chen, L. Liu, Y. Y. Peter, S. M. Samuel, Science, 2011, 331(6018), 746-750.

10 L. Liu, Y. Y. Peter, X. Chen, S. S. Mao, D. Z. Shen, Physical Review Letters, 2013, 111(6) 065505.

11 T. Leshuk, R. Parviz, P. Everett, H. Krishnakumar, R. A. Varin and F. Gu, ACS Applied Materials \& Interfaces, 2013, 5(6), 1892-1895.

12 A. Naldoni, M. Allieta, S. Santangelo, M. Marelli, F. Fabbri, S. Cappelli, C. L. Bianchi, R. Psaro and V. D. Santo, Journal of the American Chemical Society, 2012, 134(18), 7600-7603.

13 E. M. Samsudin, S. B. A. Hamid, J. C. Juan, W. J. Basirun, A. E. Kandjani, Applied Surface Science, 2015, 359, 883-896.

14 Z. Wang, C. Yang, T. Lin, H. Yin , P. Chen, D. Wan, F. Xu , F. Huang, J. Lin, X. Xie and M. Jiang, Advanced Functional Materials, 2013, 23(43), 5444-5450.

15 C. C. Chuang, C. K. Lin, T. T. Wang, V. Srinivasadesikan, P. Raghunath, M. C. Lin, RSC Advances, 2015, 5(99), 81371-81377.

16 H. Tan, Z. Zhao, M. Niu, C. Mao, D. Cao, D. Cheng, P. Feng and Z. Sun, Nanoscale, 2014, 6(17), 10216-10223. 
17 A. L. Linsebigler, G. Lu, J. T. Yates, Chemical reviews, 1995, 95(3), 735-758.

18 L. Liu, X. Chen, Chemical Reviews, 2014, 114(19), 9890-9918.

19 A. Kudo, Y. Miseki, Chemical Society Reviews, 2009, 38(1), 253-278.

20 K. Huang, X. Xie, Semiconductor Physics, P349.

21 M. S. Lazarus, T. K. Sham, Chemical Physics Letters, 1982, 92(6), 670-674.

22 Z. Zheng, B. Huang, J. Lu, Z. Wang, X. Qin, X. Zhang, Y. Dai, M. H. Whangbo, Chemical Communications, 2012, 48, 5733-5735.

23 L. Li, H. W. Tian, F. L. Meng, X. Y. Hu, W. T. Zheng, C. Q. Sun, Applied Surface Science, 2014, 317, 568-572.

24 F. M. F. De Groot, J. C. Fuggle, B. T. Thole, G. A. Sawatzky, Physical Review B, 1990, 41(2), 928.

25 F. M. F. De Groot, J. Faber, J. J. M. Michiels, M. T. Czyiyk, M. Abbate, and J. C. Fuggle, Physical Review B, 1993, 48(4), 2074.

26 M. A. Henderson, W. S. Epling, C. H. F. Peden, L. P. Craig, The Journal of Physical Chemistry B, 2003, 107(2), 534-545.

27 Diebold, U, Surface Science Reports, 2003, 48, 53.

28 A. Janotti, J. B. Varley, P. Rinke, N. Umezawa, G. Kresse, and C. G. Van de Walle, Physical Review B, 2010, 81(8), 085212.

29 D. Cronemeyer, Physical Review B, 1959, 113, 1222-1226.

30 X. Chen, L. Liu, Z. Liu, M. A. Marcus, W. C. Wang, N. A. Oyler, M. E. Grass, B. Mao, P. A, Glans, P. Y. Yu, J. Guo and S. S. Mao, Scientific Reports, 2013, 3, 1510. 


\section{Figure captions}

Figure. 1 (a) TEM image of P25 nanoparticles; HRTEM images and structural analysis of (b) pristine P25 nanoparticles, hydrogenated nanoparticles for (c) $40 \mathrm{~min}$ and (d) $80 \mathrm{~min}$.

Figure. 2 The UV-Vis absorption spectra of the pristine $\mathrm{P} 25$ and hydrogenated $\mathrm{TiO}_{2}$ treated at $300{ }^{\circ} \mathrm{C}$ for $20 \mathrm{~min}, 40 \mathrm{~min}, 60 \mathrm{~min}, 80 \mathrm{~min}$, respectively. The inset shows photographs of the five samples.

Figure. 3 (a) Schematic graph of $\mathrm{TiO}_{2}$ before and after hydrogenation; ZPS of (b) the Ti $2 p_{3 / 2}$ XPS spectra and (c) O $1 s$ XPS spectra. The insets show the corresponding raw spectra of P25 and hydrogenated $\mathrm{TiO}_{2}$ (40min). (d) Valence band spectra of the hydrogenated $\mathrm{TiO}_{2}(40 \mathrm{~min})$ with respect to the raw $\mathrm{P} 25 \mathrm{TiO}_{2}$.

Figure. 4 (a)The schematic diagram of XAS; (b) XAS of Ti $2 p$; (c) peak area ratio of the Ti $2 p$, including $e_{\mathrm{g}}$ and $t_{2 \mathrm{~g}}$ of $\mathrm{L}_{3}$ and $\mathrm{L}_{2}$.

Figure. $5{ }^{1} \mathrm{H}$ NMR spectra of hydrogenated $\mathrm{TiO}_{2}$ nanoparticles compared with raw $\mathrm{TiO}_{2}$ nanoparticle.

Figure. 6 The SPV spectra of the $\mathrm{P} 25$ and treated $\mathrm{TiO}_{2}(40 \mathrm{~min})$ under zero bias. 
Fig. 1

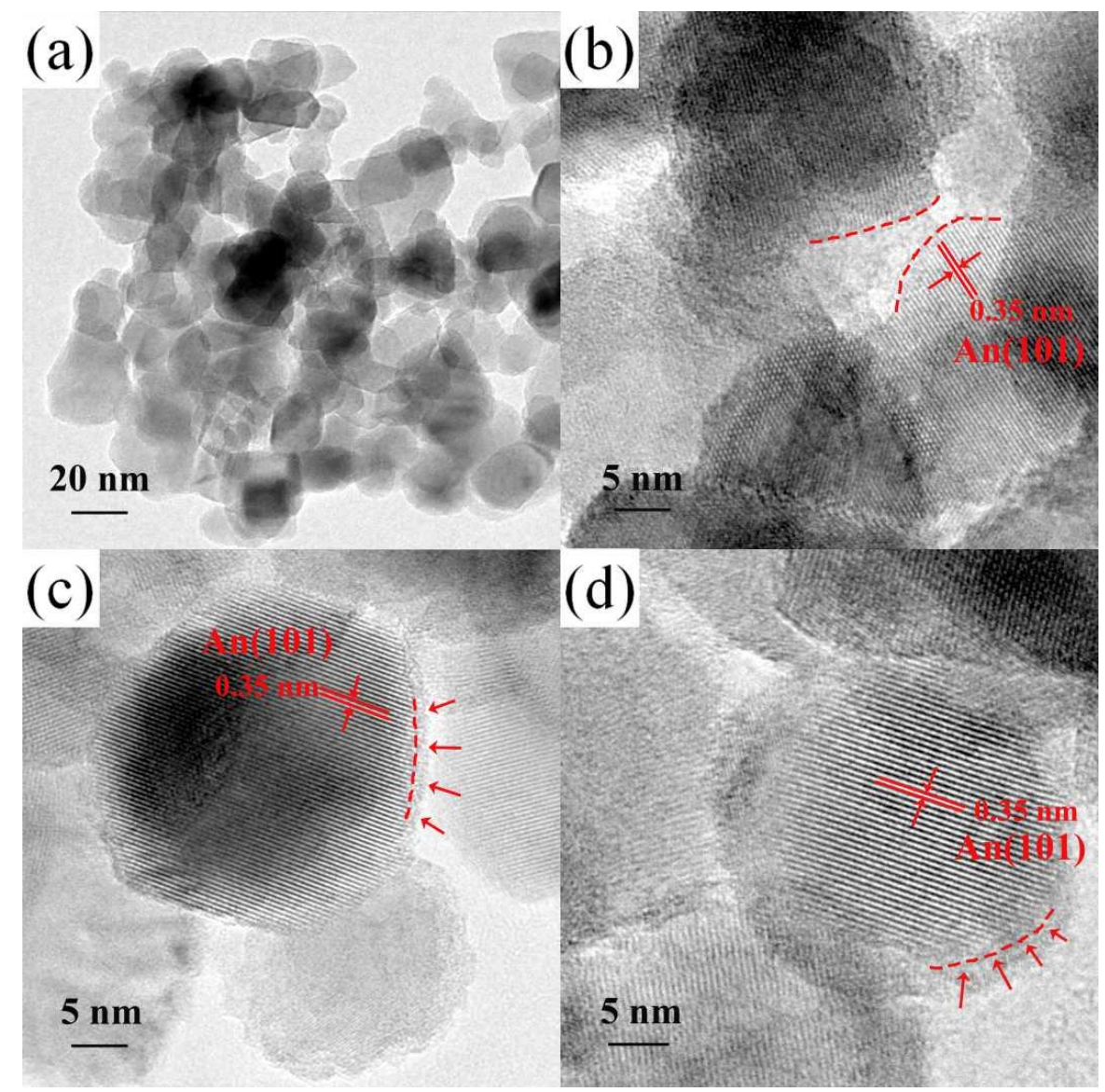


Fig. 2

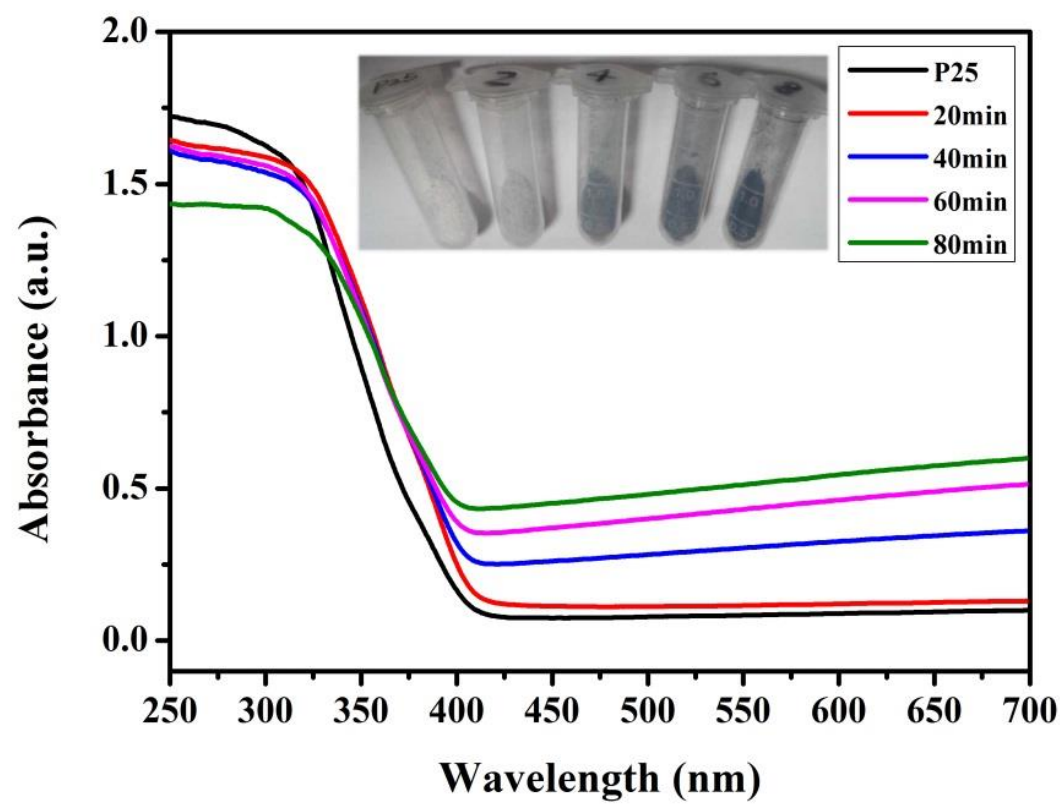


Fig. 3
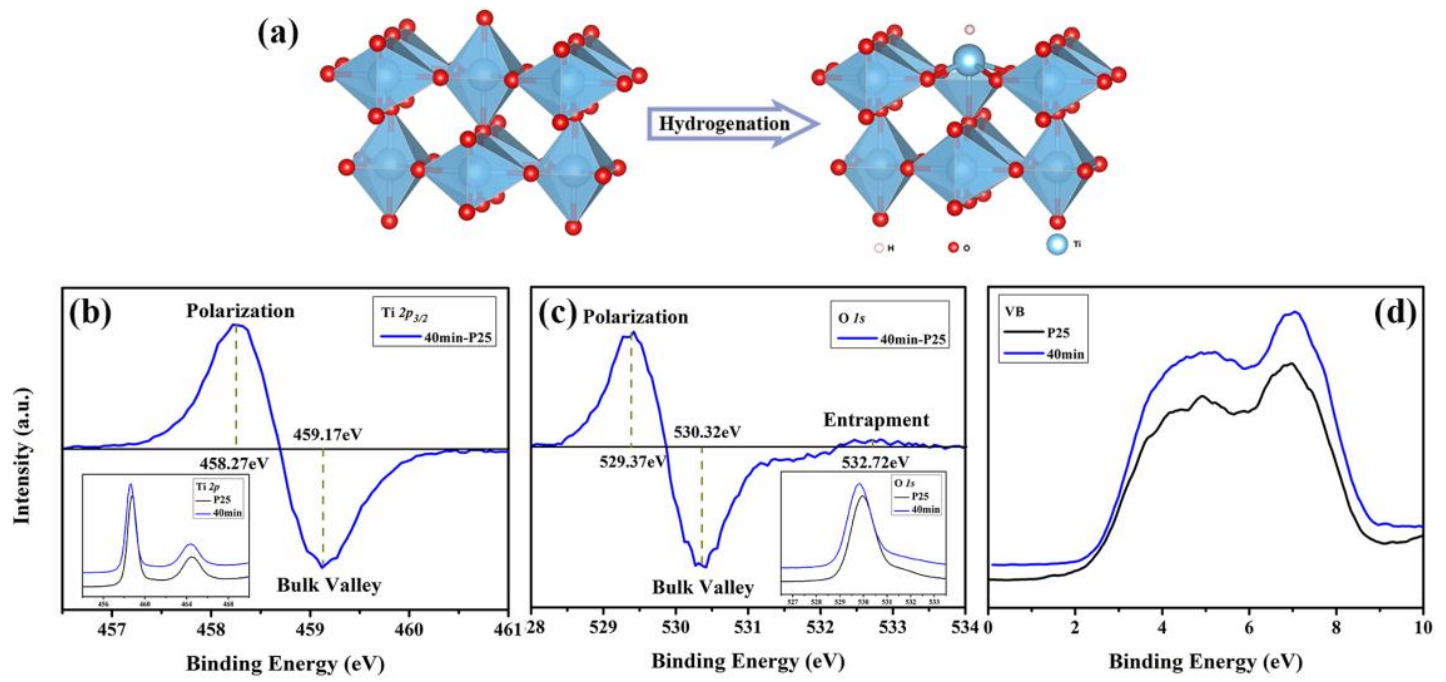
Fig. 4

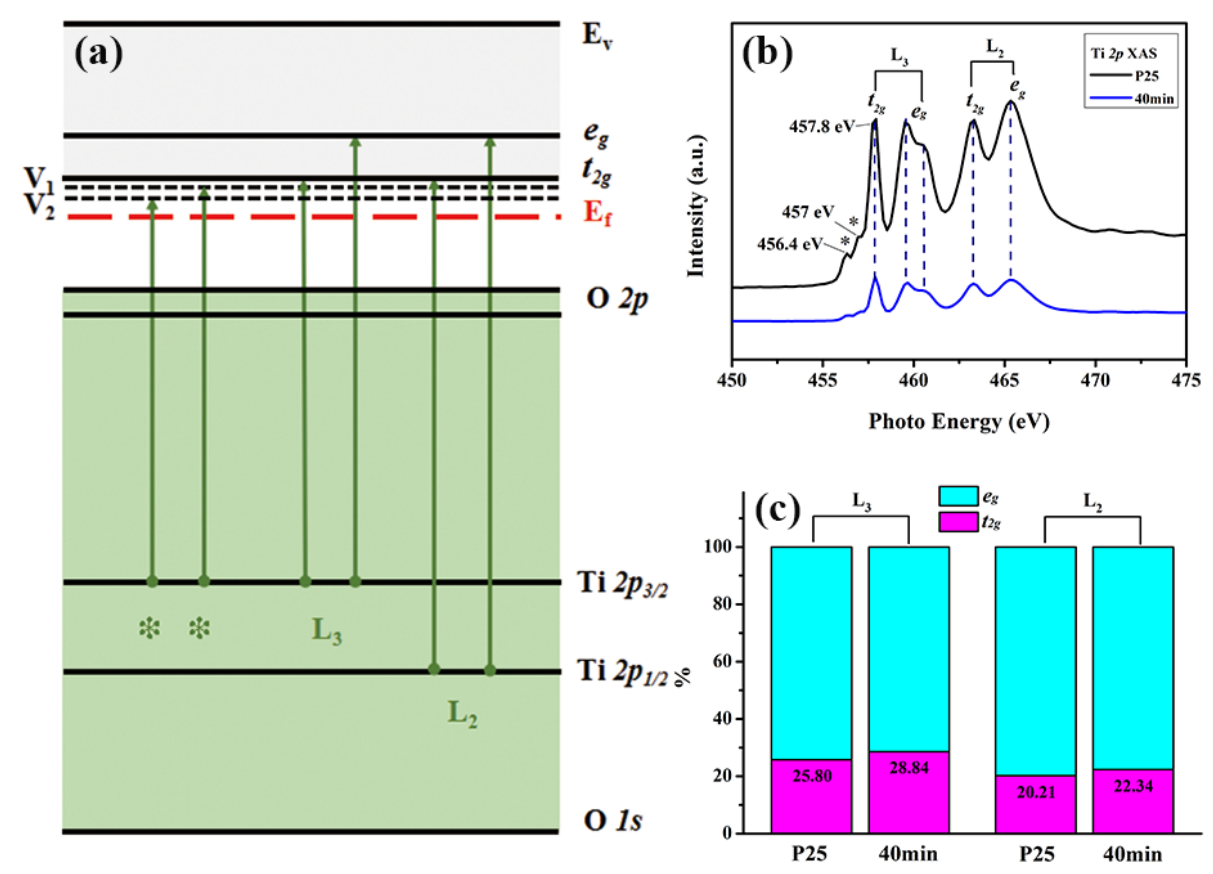


Fig. 5

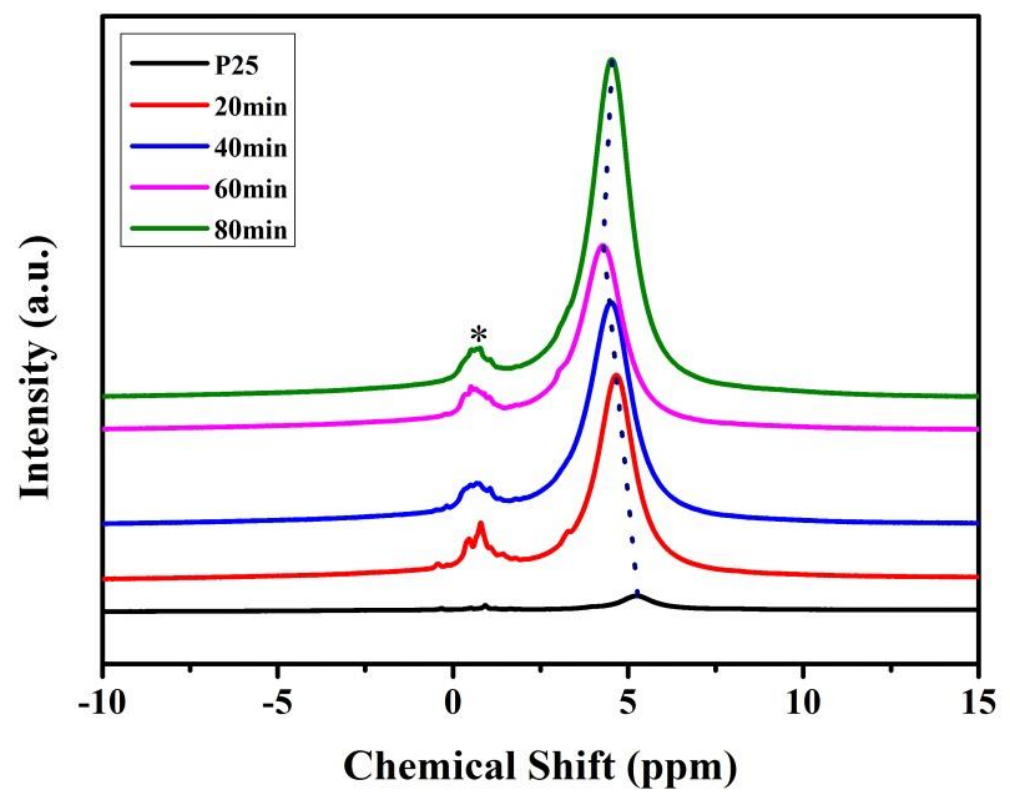


Fig. 6

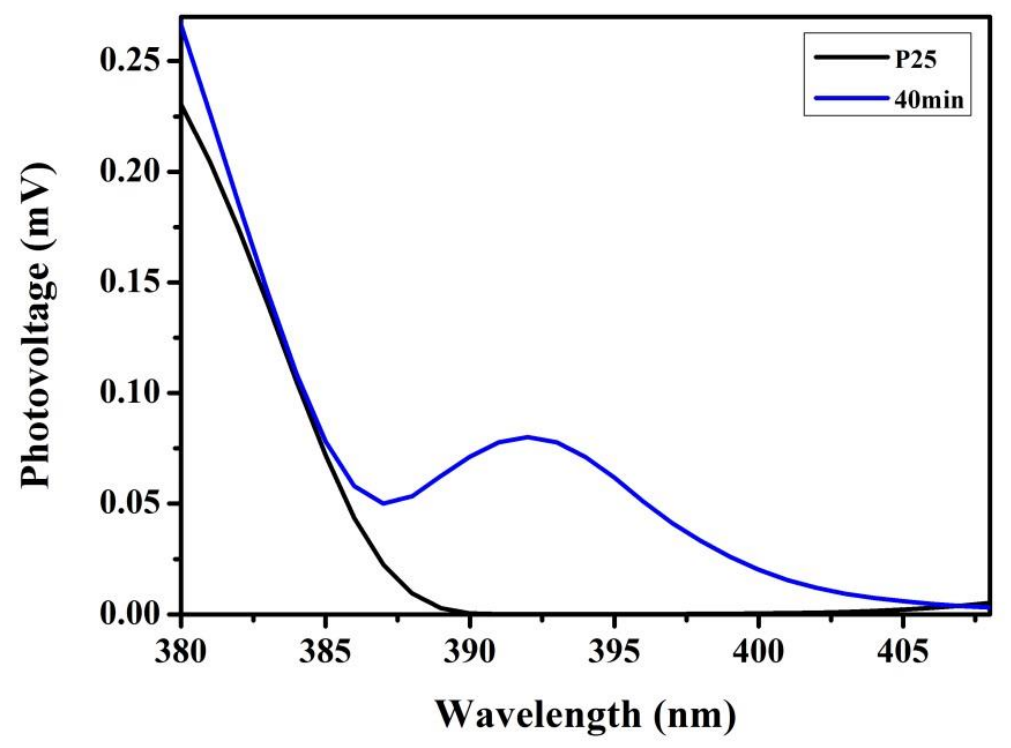

